\author{
AGNIESZKA BŁAŻEK \\ Uniwersytet im. Adama Mickiewicza w Poznaniu \\ blazek@amu.edu.pl \\ ORCID: 0000-0002-4741-0985
}

\title{
Kommunikative Anstrengung. Hin zur lebensnahen interkulturellen (Fremdsprachen-)Lehrerausbildung
}

\author{
Communicative effort. \\ Toward a true to everyday life intercultural \\ (foreign language) teacher training
}

\begin{abstract}
Intercultural (foreign language) teacher training tends to concentrate on selective case studies developed to show communicative problems, caused by their very special intercultural and multilingual characteristics. It also tends to make a big promise of a rapid improvement in intercultural communication in a multicultural and multilingual society by transferring the solutions for intercultural communicative problems to the students' own experience. Such an approach fails to take into account the fact that encounters with inadequate communicative acts (behaviours/speech acts) determined by cultural and/or language differences are a daily experience of everybody and require a lot of communicative effort in order to prevent intercultural problems before they happen. The following article discusses what is meant by communicative effort, how past studies on the intercultural competence of (foreign language) teachers account on communicative effort, and how to prepare students to undertake communicative effort in their daily communicative activities in a multicultural and multilingual society.
\end{abstract}

KEYWORDS: multilingualism, communicative effort, intercultural communication, intercultural teacher training.

SCHLÜSSELWORTE: Mehrsprachigkeit, kommunikative Anstrengung, interkulturelle Kommunikation, interkulturelle Lehrerbildung. 


\section{EINFÜHRUNG}

Interkulturelle Kommunikation und interkulturelle Kompetenz sind Schlagwörter, die seit Jahren fest im Umkreis der Forschung zur Lehrerausbildung, darunter auch der Fremdsprachenlehrerausbildung etabliert sind. Dies verwundert nicht, denn die Erfahrung einer multilingualen und multikulturellen Gesellschaft - von der Schulbank über die globale Vernetzung von Unternehmen bis hin zu globalen Social Media - ist so alltäglich geworden, dass sie auch im Rahmen von Schul- und Hochschulsystemen vorbereitet und begleitet werden muss.

Interkulturelle Ausbildungsinhalte gehen oft von kritischen (und dadurch auch negativ behafteten) Situationen eines Aufeinanderstoßens von Kommunikationsteilnehmern unterschiedlicher Kulturen (und oft anderer Sprachen) aus (z. B. critical incidents, Kulturschock, Vorurteile). Diese werden meistens als (realitätsnahe) Fallbeispiele angeboten und mit der Hoffnung interpretiert und bearbeitet, dass die Lernenden die (selbst ausgearbeiteten) plausiblen Lösungsvorschläge internalisieren, diese bei auftretenden kultur- und sprachbedingten Problemen umsetzen und dadurch interkulturelle Probleme lösen können. Als Ziel gilt die Entwicklung einer interkulturellen Kompetenz, mit der positiv konnotierte Teilziele erreicht werden, darunter die Offenheit, die Bereitschaft, eigene Werte zu relativieren und sich konstruktiv mit der Fremdheit auseinanderzusetzen. Höchstens der Ausdruck Ambiguitätstoleranz könnte vermuten lassen, interkulturelle Kompetenz schließt auch Fähigkeiten ein, die nicht immer das Verbleiben in einer kulturellen und sprachlichen Komfort- und Zufriedenheitszone garantieren. Ambiguitätstoleranz würde nämlich bedeuten, kulturelle/sprachliche Mehrdeutigkeiten, Unsicherheiten grundsätzlich aushalten (tolerieren) zu können, (wahrscheinlich) ohne sie immer rational nachvollziehen zu können.

Dies versteht sich weder als ein Plädoyer für die Neu- bzw. Umbenennung von Begriffen der Interkulturalität noch als ein Aufruf zur Neudefinition von interkulturellen Ausbildungsinhalten. Allerdings sollte darauf hingewiesen werden, dass Lernenden meist kaum bewusst gemacht wird, mit welchem Aufwand auf Seiten eines jeden der beteiligten Kommunikationsteilnehmer die Umsetzung interkultureller Ziele verbunden ist. Es ist ein Aufwand, der tagtäglich, immer von Neuem und ohne Aussicht auf einen endgültigen Abschluss zu leisten ist. Gemeinschaften (seien es kulturelle, sprachliche oder fachliche) entwickeln im Zuge der Interaktion (darunter der Kommunikation) Handlungsroutinen, Schemata usw., die es ihnen erleichtern, gemeinsame (bzw. gemeinsam vereinbarte) gesellschaftliche, fachliche usw. Ziele umzusetzen. Der Umgang mit als gegenüber diesen Handlungsroutinen, Schemata usw. inadäquat empfundenen (kulturellen, 
sprachlichen aber z.B. auch fachlichen/fachsprachlichen) Handlungen (Inkongruenzen) ist - auch wenn wir große interkulturelle Ziele vor Augen haben - schlicht und ergreifend anstrengend. Diese Anstrengung ist dabei nicht nur in Situationen gefragt, die die interkulturelle Forschung als hotspots/rich points (Agar 1994) identifiziert - sie ist in einer jeder Situation einer kulturellen/sprachlichen Inadäquatheit gefragt und lässt sich allein dann reduzieren, wenn diese Inadäquatheiten in gleichen/ähnlichen Formen häufiger und regelmäßiger auftreten (und oft auch zur Routine werden) ${ }^{1}$.

Im Folgenden möchte ich:

- zwei Fallbeispiele vorstellen, an denen ich konkret bespreche, was unter kommunikativer Anstrengung verstanden wird,

- zwei Studien zur Entwicklung bzw. Evaluierung der interkulturellen Kompetenz vorstellen, an deren Revision ich die Relevanz der kommunikativen Anstrengung für den Bereich interkulturelle (Fremdsprachen-)Lehrerausbildung synthetisch nachweise,

- Anstöße für einen didaktischen Umgang mit der kommunikativen Anstrengung formulieren.

\section{FALLBEISPIELE}

\section{Fallbeispiel 1}

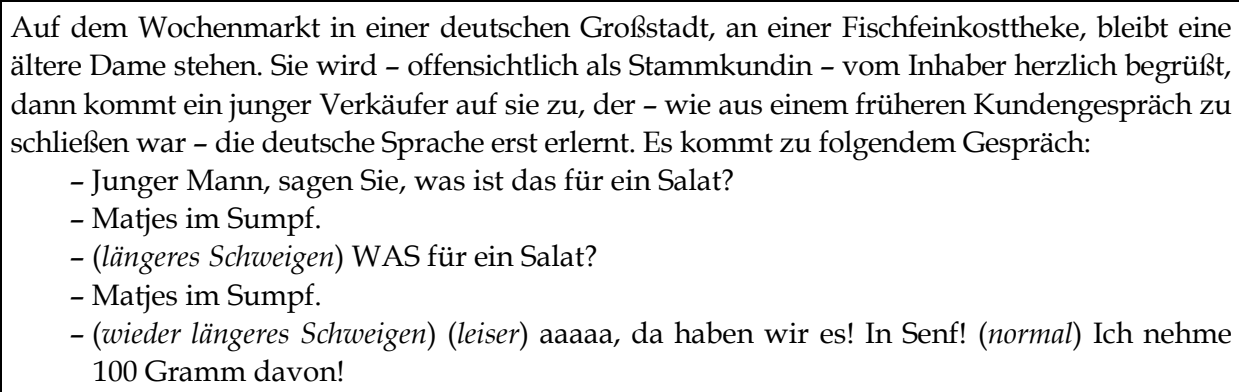

${ }^{1}$ Ein gutes Beispiel dafür wäre die Toleranz für Akzentinadäquatheiten, die ich am eigenen Beispiel veranschaulichen möchte. Als Polin, die kaum mit nichtmuttersprachlichen Sprechern der polnischen Sprache spricht, hatte ich in Deutschland Probleme, meine exzellent Polnisch, doch mit hörbarem ,deutschem Akzent' sprechenden Studierenden (der Polenstudien) verstehen zu können. Dieses Problem ist nach einigen Seminaren vollständig verschwunden. Da sich innerhalb einiger Tage der Akzent der Studierenden nicht wesentlich verändert haben wird, konnte ich davon ausgehen, dass meine Toleranz für die Akzentinadäquatheit gestiegen ist. In ähnlicher Weise beobachte ich, dass die Toleranz muttersprachlich deutschsprachiger Kollegen, die in einer multikulturellen Gesellschaft leben und arbeiten, für verschiedene (meistens muttersprachlich bedingte) Akzentinadäquatheiten im Deutschen viel größer ist als bei mir, die ich Deutsch als Fremdsprache spreche und grundsätzlich in Polen das sog. Hochdeutsch erlernt habe. 
Die Kundin produziert eine konversationelle Reparatur („In Senf!“), die sich allerdings nicht als Fehlerkorrektur versteht, sondern als lautes Denken. Der Fehler liegt im Bereich ihrer Fehlertoleranz, denn aus dem Kontext heraus schließt sie dank ihrer interpretativen Kompetenz, dass es sich nicht um "Sumpf" handeln kann, sondern um ein akustisch ähnliches Wort. Außerdem war sie durch den Verlauf des vorausgehenden Kundengesprächs schon darauf eingestellt, dass eine volle kommunikative Adäquatheit der Äußerungen des Verkäufers eventuell nicht vorausgesetzt werden kann. Sie versucht, ein mit "Sumpf“ akustisch verwandtes Wort zu finden, das sie in den Kontext sinnvoll einfügen könnte. Dies gelingt ihr. Sie merkt und lobt den eigenen Denkaufwand, indem sie sagt ", da haben wir es!“.

\section{Fallbeispiel 2}

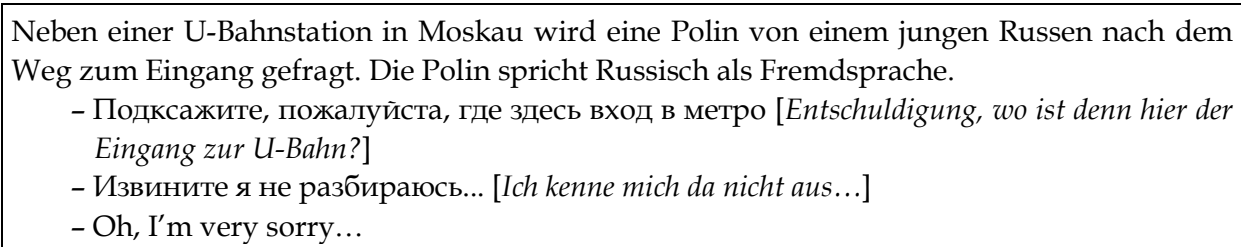

Es kommt hier zu einem Code-Switching. Damit wird darauf verwiesen, dass eine als allgemeingültig angesehene Konvention offensichtlich verletzt wurde. Der Russe war darauf eingestellt, eine den Adäquatheitskriterien entsprechende Antwort zu bekommen. Die Nichterfüllung der pragmatischen Adäquatheitskriterien (Извините я не разбираюсь... Mögliche Varianten: идите на право, на лево, вот здесь, я не отсюда), womöglich gekoppelt mit einem heraushörbaren fremden Akzent, führt ihn zu dem Schluss, dass er die erwartete Antwort nicht bekommen kann und er akzeptiert die Gründe dafür: Er hat es offensichtlich mit einer des Russischen eingeschränkt mächtigen Ausländerin zu tun. Durch Code-Switching weist er darauf hin, dass er den Aufwand des Verstehens und der Wertung durchlaufen hat und er bringt dies durch den Sprachwechsel zum Ausdruck.

Diese zwei Situationen sind keine sog. Critical Incidents, auch keine Hotspots/bzw. Rich Points. Es handelt sich also nicht um Situationen, die einen interkulturellen Konflikt beschreiben (wobei sie aufgrund der jeweiligen Fehler der Produzenten womöglich das Potenzial dazu hätten). Viel mehr würde ich sie als alltägliche Interaktionen in einer mehrsprachigen und multikulturellen Gesellschaft bezeichnen, die den Aufwand veranschaulichen, den alle Kommunikationspartner in eine auf Mehrsprachigkeit beruhende Verständigung investieren müssen. Der junge Verkäufer an der Fischkosttheke 
und die Polin sind in diesem Fall jeweils diejenigen, die versuchen aufgrund ihrer erlernten Sprachressourcen einen in grammatischer und pragmatischer Hinsicht als adäquat empfunden Text zu produzieren. Ihre Leistung ist hier hauptsächlich eine Sprachlern- und -anwendungsleistung. Die ältere Dame und der junge Russe, für die die Kommunikation auf der Grundlage eines breit geteilten (intersubjektiven) Wissens und einer weithin gemeinsamen Sprache weitgehend ein unaufwendiges, leichtläufiges Verfahren zur Erreichung ihrer Handlungsziele ist, werden aus ihrer Routinegewissheit gebracht und zur Reflexion bzw. zur flexiblen Entwicklung neuer kommunikativen Verfahren herausgefordert. Ihre Leistung ist in diesem Fall hauptsächlich eine Adaptierungsleistung, die in der Bearbeitung der Erfahrung der Inkongruenz besteht.

Die hier geschilderten kommunikativen Leistungen, ja den kommunikativen Aufwand auf beiden Interaktionsseiten, nenne ich, wie eingangs beschrieben, kommunikative Anstrengung (vgl. Garfinkel 2006 [1948]). Ohne den Anspruch, eine neue Perspektive für die interkulturelle Kommunikation aufmachen zu wollen, möchte ich damit darauf hinweisen, dass die kommunikative Anstrengung meiner Ansicht nach generell für alltägliche Interaktionen in einer auf Mehrsprachigkeit basierenden, interkulturellen Kommunikation charakteristisch ist. Hierbei handelt sich allerdings nicht nur um eine kommunikative Anstrengung derjenigen, die den Aufwand betreiben, sich einer fremden Sprache in einer fremdkulturellen Gesellschaft (Gemeinschaft) zu bedienen, sondern auch der derjenigen, die in der muttersprachlichen und für sie gewöhnlichen Interaktion auf Inkongruenzen stoßen.

Der Begriff der kommunikativen Anstrengung wird hier aus der Ethnomethodologie des Soziologen Harold Garfinkel (ebd.) abgeleitet, durch einige Gedanken von Clark (1996) zu Wissenskommunikation ergänzt und insgesamt auf den interkulturellen Kontext angewandt. Garfinkels Forschungsinteresse bezog sich auf alltägliche Situationen von Wissensgemeinschaften (Organisationen). Er ging davon aus, dass der Alltag dieser Gemeinschaften nicht "selbstverständlich", sondern gemäß Regeln funktioniert, die nirgendwo verbindlich festgeschrieben sind und trotzdem durch die Mitglieder einer konkreten Gemeinschaft in konkreten Situationen angewandt werden. Die Regeln sind allerdings keine Anweisungen, denen die Teilnehmer einer konkreten Situation blind folgen, sondern sie sind Eigenschaften von Situationen, in die Teilnehmer eintreten. Sobald Teilnehmer auf eine Situation treffen, gestalten sie ihre Handlungen so, dass sie an den vorgefundenen Kontext anschließen und ihn fortführen. Die Regeln werden allerdings für die Teilnehmer einer Situation erst dann sichtbar, wenn sie auf Inkongruenzen stoßen, d. h. wenn einer der Teilnehmer in einer Situation inadäquat handelt (Garfinkel 1967). Garfinkel (ebd.) war der Meinung, dass Teilnehmer 
von Situationen in der Lage sind, bei Inkongruenzen (d. h. bei inadäquaten Handlungen anderer Teilnehmer) ihre Handlungen $\mathrm{zu}$ begründen und $\mathrm{zu}$ rechtfertigen (account for). Bei der Begründung (die als kommunikative Handlung ausgeführt wird) greifen sie nämlich auf genau denselben Wissensvorrat über die Situation zurück, wie bei der Ausführung der Handlungen selbst (d.h. auf das Wissen, das sie in eine Situation einbringen, um in dieser handlungsfähig zu bleiben und als kompetente Teilnehmer angesehen $\mathrm{zu}$ werden). Diesen reflexiven Umgang mit den eigenen Handlungen und die Fähigkeit, sie zu begründen, nennt Garfinkel kommunikative Anstrengung (2006 [1948]).

Um empirischen Zugang zu den Regeln von Alltagssituationen zu erhalten, hat Garfinkel sog. Krisenexperimente durchgeführt (Garfinkel 1967). Der Sinn dieser Experimente war es, Situationen herbeizuführen, die die Selbstverständlichkeit des Alltags stören. Erst die Herbeiführung einer Störung (Krise) in einer banalen Situation würde es erlauben, Selbstverständlichkeiten nachzuvollziehen. Garfinkel war somit nicht daran gelegen, eine wissenschaftliche Methode der Post-hoc-Interpretation von Regeln des Alltagslebens zu formulieren. Viel mehr war er daran interessiert, dazu anzuregen, Ad-hoc-Erklärungen für die Handlungen von Teilnehmern an ausgewählten Alltagssituationen zu formulieren, um dadurch zu praktischen Lösungen zu kommen. Namentlich war ihm an solchen Lösungen gelegen, die Menschen im Alltag heranziehen, um bestimmte Handlungen trotz Störungen fortführen zu können.

Die Frage, die sich an dieser Stelle aufdrängt, lautet: Ist in der Alltagskommunikation eine solche (permanente) kommunikative Anstrengung, wie sie Garfinkel beschreibt, überhaupt notwendig? Müssen Menschen in ihrem Alltag mit ihren Handlungen (darunter Kommunikationshandlungen) stets reflexiv umgehen? Warum sollten sich Mitglieder der jeweiligen Gemeinschaften/Teilnehmer von Situationen der Regeln ihres Alltags bewusst werden und diese rekonstruieren? Zur Beantwortung dieser Frage ziehe ich die psycholinguistische Kommunikationstheorie von Clark (1996) heran.

Während Garfinkel erläutert, dass Menschen bei der Ausführung (und Begründung) ihrer Handlungen in einer konkreten Situation auf einen Wissensvorrat zurückgreifen, der aus Informationen über die Merkmale dieser Situation und die entsprechend in ihr anzuwendenden Regeln besteht, ist Clark (1996) in Bezug auf die Kommunikation der Meinung, es handle sich bei dem Wissensvorrat um eine Schnittmenge der individuellen Wissensvorräte aller Teilnehmer einer Kommunikationssituation, $d . h$. um einen ihnen gemeinsamen kognitiven Referenzrahmen, den sog. Common ground (geteilten Wissenshintergrund), der lokal (d.h. stets in der jeweiligen Situation) von den Kommunikationsteilnehmern „ermittelt" wird. Dies geschehe derart, 
dass im Zuge der Kommunikation seitens der Teilnehmer Annahmen (Hypothesen) über den individuellen Wissensbestand der Kommunikationspartner - d. h. über ihr Vorwissen, ihren sozialen Status und ggf. ihr Fachwissen, die von ihnen wahrgenommenen Situationsinhalte, den früheren Gesprächsverlauf usw. - gemacht und diese anhand der Reaktionen (Signale) der Partner verifiziert werden. Je größer der (angenommene) gemeinsame Wissenshintergrund ist, desto mehr sind die Kommunikationspartner geneigt, der Situation entsprechende konventionelle (sprachliche) Kommunikationshandlungen (sprachliche Routinen, formelhafte/schematische Ausdrü$\mathrm{cke}^{2} \mathrm{usw}$.) einzusetzen. Dies geschieht mit dem Ziel, ihre Kommunikationsintentionen mit dem geringsten kommunikativen Aufwand realisieren zu können. Ist der (angenommene) gemeinsame Wissenshintergrund klein, so erweist sich das Zurückgreifen auf Routinen als unmöglich bzw. ist nur eingeschränkt oder aber erst nach der Erweiterung des gemeinsamen Wissenshintergrunds (z. B. durch Übermittlung entsprechender Informationen über die Kommunikationssituation) möglich. Damit steigt allerdings der kommunikative Aufwand der Gesprächspartner an.

Laut der Theorie von Clark würden also Kommunikationsteilnehmer ihre Kommunikationssituationen immer rational steuern sowie ihre kommunikative Anstrengung reduzieren bzw. steigern können, in Abhängigkeit von ihrer Hypothese über den gemeinsamen Wissenshintergrund der Kommunikationsteilnehmer. Fraglich bleibt jedoch, a) ob die Teilnehmer einer Kommunikationssituation immer imstande sind, diese Hypothesen angemessen zu stellen (d.h. Signale über die Kommunikationssituation angemessen zu interpretieren) und b) ob sie immer dem angenommenen gemeinsamen Wissenshintergrund adäquat handeln (wollen) (d. h. ob sie z. B. trotz eines vermuteten geringen gemeinsamen Wissenshintergrunds auf Kommunikationsroutinen zurückgreifen).

Nach diesem kurzen Exkurs möchte ich auf die eingangs gestellte Frage nach dem Sinn der kommunikativen Anstrengung zurückkommen. Vor dem Hintergrund der Ausführungen von Clark steht nämlich außer Frage, dass das Prinzip des minimalen kommunikativen Aufwands Kommunikationsteilnehmern dabei hilft, die Alltagskommunikation effizienter zu gestalten. Durch den (unreflektierten) Rückgriff auf Kommunikationsroutinen und -schemata reduzieren sie legitimerweise ihren kommunikativen Aufwand und bewirken, dass ihre eigenen Ziele (und womöglich die Ziele der jeweiligen Gemeinschaft, in der sie ihre Kommunikationshandlungen realisieren) schneller umgesetzt werden. Sollten die Kommunikationsteilnehmer gegen

2 In der Linguistik, insbesondere in der Fachsprachenlinguistik wird dieses Problem unter dem Stichwort sprachliche Konventionen erforscht (vgl. z. B. Błażek 2018: 137-142). 
dieses Prinzip „verstoßen“, d. h. sollten sie reflektiert mit den eigenen (kommunikativen) Alltagsroutinen umgehen und eine kommunikative Anstrengung vornehmen, so muss ihnen bewusst werden, dass gerade der Verzicht auf Alltagsroutinen in einer konkreten Kommunikationssituation zur Effektivierung der Kommunikation führt. Dies kann ihnen bewusst werden, wenn sie

- in der Lage sind, Signale in der Kommunikationssituation adäquat zu interpretieren und $\mathrm{zu}$ angemessenen Schlussfolgerungen hinsichtlich des gemeinsamen Wissenshintergrunds der Kommunikationsteilnehmer zu kommen,

- auf die Bequemlichkeit einer mechanischen Anwendung von Konventionen verzichten wollen ${ }^{3}$.

Will man die vorstehenden Ausführungen auf Prozesse interkultureller Kommunikation in mehrsprachigen Gesellschaften übertragen, so liefern sie die folgenden Anhaltspunkte:

- in einer Kommunikationssituation, in der einer der Teilnehmer eine Fremdsprache bzw. Zweitsprache spricht, sind alle beteiligten Personen stets einer Erfahrung von Inkongruenz ausgesetzt und es bedarf somit stets kommunikativer Anstrengung (des Begründen und Rechtfertigens eigener Kommunikationshandlungen),

- die auftretenden Inkongruenzen sind grundsätzlich nur durch diejenigen Teilnehmer der Kommunikation nachvollziehbar und begründbar, die ihre Muttersprache sprechen; Teilnehmer, die in der besagten Situation eine Fremdsprache oder Zweitsprache sprechen, können eine unbewusst hervorgerufene Störung des (für die Kommunikation in einer konkreten Sprache) üblichen Kommunikationsverlaufs lediglich an den Kommunikationssignalen (d. h. an den Reaktionen anderer Kommunikationsteilnehmer) ablesen - für sie beruht die kommunikative Anstrengung einerseits darin, die für die Kommunikation in einer Fremdsprache/Zweitsprache charakteristischen Routinen einhalten zu müssen und andererseits die für sie charakteristischen Kommunikationssignale angemessen zu interpretieren;

- durch die Wiederholung von Kommunikationssituationen ist das Begründen und Rechtfertigen eigener Kommunikationshandlungen auch in einer Fremd- bzw. Zweitsprache erlernbar,

${ }^{3}$ Mit dem Verb „wollen“ werden Einstellung bzw. Intentionen der Kommunikationsteilnehmer angesprochen. Auch wenn sie ihre Kommunikation grundsätzlich nach dem Prinzip der Kommunikationseffizienz ausrichten (sollten), ist es durchaus denkbar, dass sie es nicht tun wollen. Auch umgekehrt ist denkbar, dass die Bequemlichkeit der mechanischen Regelanwendung nicht aus Effizienzgründen aufgegeben wird, sondern um z.B. den Alltag nicht "einfach" hinzunehmen, sich in einer (gesellschaftlichen) Rolle durch Verwendung sprachlicher Schemata intentional nicht festzulegen bzw. festlegen zu lassen, um einen Novizen in eine Fachgemeinschaft einzuführen, einen Laien über komplexe Fachinhalte zu informieren usw. 
- eine sich in einer Sprachgemeinschaft wiederholende und auf die Verwendung der Fremdsprache/Zweitsprache zurückzuführende Inkongruenz in der Kommunikation kann zur Gewohnheit werden. Dadurch wird letztlich die kommunikative Anstrengung reduziert.

Nach dieser theoretischen Einführung soll nachstehend anhand von zwei evaluativen Studien, die vollständig bzw. teilweise im Bereich der interkulturellen Kompetenz verankert sind, gezeigt werden, ob und wenn ja, welche Bedeutung dem Phänomen der Anstrengung in der einschlägigen empirischen Forschung zur interkulturellen Kommunikation zukommt. Die Studien setzen implizit unterschiedliche Typen von Mehrsprachigkeit voraus - während die erste Studie eher die schulische, erlernte Mehrsprachigkeit impliziert, behandelt die zweite lebensweltliche, erworbene Mehrsprachigkeit. Beide Studien liegen ca. 10 Jahre zurück und wurden unter Bedingungen durchgeführt, die heute nur noch zum Teil gelten können. Dies bezieht sich vor allem auf die Studie zur Integration von Aussiedlerkindern in Schulen Niedersachsens und Sachsen-Anhalts. Trotzdem greife ich auf sie zurück, denn die gesammelten Daten werfen ein Licht auf das hier interessierende Phänomen der Anstrengung, das in der früheren Datenauswertung als Faktor nicht berücksichtigt wurde.

\section{STUDIE NR. 1}

Evaluation interkultureller Kompetenz, Beispiel: Polen (Błażek 2008)

Es handelt sich hier um ein Forschungsprojekt, das zum Ziel hatte, die nach 1990 auf interkulturelles Lernen und Lehren umorientierte Ausbildung von Lehrenden für Deutsch als Fremdsprache in Polen auf ihre Effektivität zu evaluieren. Die Evaluation erfolgte prozess- und ergebnisorientiert (Niemierko 1999). Zum einen sollte über die Analyse von Dokumenten aus 7 Deutschlehrerausbildungseinrichtungen in Polen herausgefunden werden, inwiefern in der Bestimmung der Lernziele, der Auswahl der Lerninhalte und Lernverfahren das Anliegen der interkulturellen Bildung verfolgt wird. Der Analyse wurden Lehrpläne und -inhalte offiziellen und öffentlichen Charakters unterzogen. Zum anderen sollte mittels eines Fragebogens, dessen Kern ein Selbstevaluationsbogen und eine Critical-Incident-Aufgabe darstellten, das Niveau der interkulturellen Kompetenz von Lehramtsstudierenden für Deutsch als Fremdsprache in Polen punktuell ermittelt werden. Insgesamt wurden 180 polnische Studierende mit Deutschkenntnissen auf einem Niveau von B2 bis C1 befragt. Die triangulatorisch angelegte empirische Untersuchung wurde durch Experteninterviews mit insgesamt 13 Do- 
zenten ergänzt, um die Schlussfolgerungen und Hypothesen der vorangehenden Teile der Untersuchung überprüfen und besser einordnen zu können.

Der Evaluation lag das in der Fremdsprachendidaktik inzwischen sehr verbreitete Modell der interkulturellen Kompetenz nach Byram (1997) zugrunde. In diesem Modell wird die interkulturelle Kompetenz im Zusammenspiel mit anderen Teilkompetenzen ausgewiesen, die für das Lernen von Fremdsprachen relevant sind und zusammen als interkulturelle kommunikative Kompetenz bezeichnet werden:

- Eine linguistische Kompetenz (Beherrschung des Sprachcodes),

- Eine soziolinguistische Kompetenz (situativer Kontext der Äußerungen, der soziale Status der Gesprächspartner etc.),

- Eine Diskurskompetenz (Texte, Textsorten und ihre Verknüpfung zum Diskurs),

- Eine interkulturelle Kompetenz.

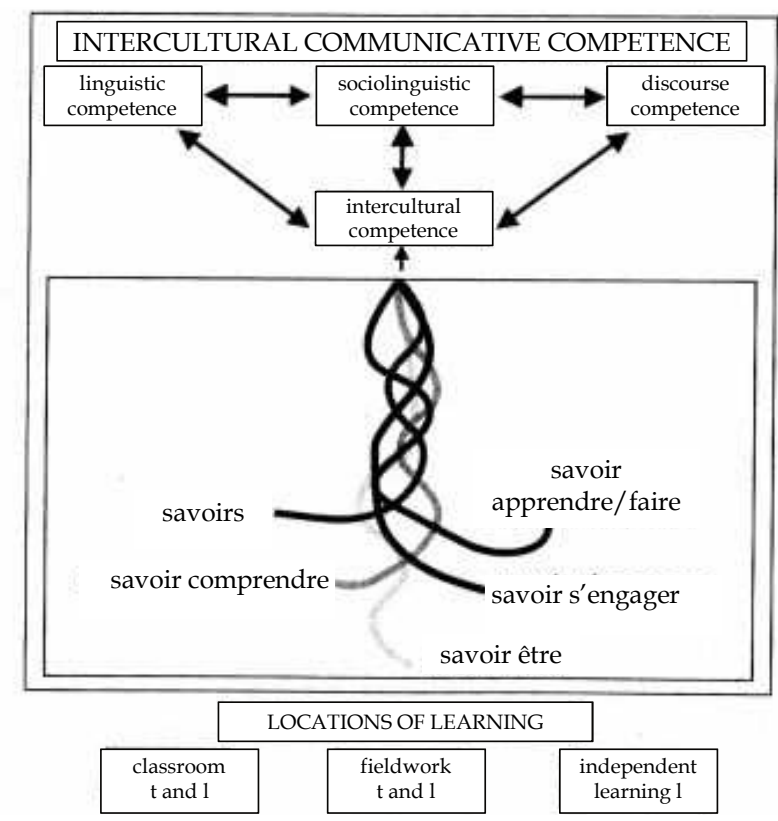

Abbildung 1. Dimensionen interkultureller kommunikativer Kompetenz nach Byram (1997: 73)

Die interkulturelle Kompetenz im engeren Sinne wird in diesem Modell weiter spezifiziert. Lies Sercu (2004) „übersetzt“ dieses Modell in die traditionelle Sprache der Lehr- und Lernziele im Fremdsprachenunterricht, die sich in drei Bestandteile gliedern: Wissen (kognitive Domäne) - Haltungen (affektive Domäne) - Fertigkeiten (skillspezifische Domäne). 
Tabelle 1. Ebenen interkultureller Kompetenz nach Sercu (2004: 74)

\begin{tabular}{|l|l|}
\hline \multicolumn{1}{|c|}{ Ebenen der IK } & \multicolumn{1}{c|}{ Allgemeine Qualifikationen } \\
\hline \multirow{5}{*}{ Wissen } & allgemeine Kulturkenntnisse \\
\cline { 2 - 3 } & Wissen über die eigene und fremde Kultur \\
\cline { 2 - 3 } Einstellungen & Kenntnisse der individuellen und sozialen Interaktion \\
\cline { 2 - 3 } & $\begin{array}{l}\text { Einsicht in die Art und Weisen, in der Sprache auf ihrer verbalen und } \\
\text { paraverbalen Ebene von Kultur beeinflusst wird }\end{array}$ \\
\hline \multirow{5}{*}{ Handlungen } & $\begin{array}{l}\text { Offenheit und Bereitschaft, die eigene Perspektive zu relativieren } \\
\text { Neugierde }\end{array}$ \\
\hline & $\begin{array}{l}\text { Interpretationsvermögen im Hinblick auf die fremde Kultur und } \\
\text { ihren Bezug auf die eigene Kultur }\end{array}$ \\
\cline { 2 - 3 } & Fähigkeit, die Realität und Interaktion zu beobachten \\
\cline { 2 - 3 } & $\begin{array}{l}\text { Fähigkeit, Kenntnisse über eine fremde Kultur zu erwerben und diese } \\
\text { in der Kommunikation und Interaktion angemessen anzuwenden }\end{array}$ \\
\cline { 2 - 3 } & $\begin{array}{l}\text { Fähigkeit, metakognitive Strategien einzusetzen: über eigenes Lernen } \\
\text { zu reflektieren }\end{array}$ \\
\cline { 2 - 3 } & $\begin{array}{l}\text { kritisches Urteilsvermögen im Hinblick auf die eigene und fremde } \\
\text { Kultur }\end{array}$ \\
\hline
\end{tabular}

Es ist nicht möglich, die Ergebnisse der Studie detailliert darzustellen, zumal als Ergebnis nur eine Tendenzbeurteilung möglich war. Deshalb gehe ich kurz auf die allgemeinen Tendenzen ein und fokussiere vor diesem Hintergrund nur einige Probleme, die mir als Impulse für das eingangs geschilderte Problem der kommunikativen Anstrengung relevant scheinen.

Die Analyse der Studienpläne, -inhalte und -methoden zeigte die generelle Präsenz eines interkulturellen Schwerpunktes. Dabei ergab sich allerdings Folgendes:

- Interkulturelle Inhalte sind meistens binational, auf die Konstellation Deutschland-Polen, angelegt. Sie enthalten nur wenige Bezüge auf andere deutschsprachige Länder. Eine universale Methodenkompetenz, mit der die Lernenden in jeder interkulturellen Situation zurechtkommen können, wird weniger trainiert.

- Die einschlägigen Themen und Methoden sind meistens aus der interkulturellen Pädagogik adaptiert, z. B. Auto- und Heterostereotype, Klischees und Vorurteile, Toleranz, das Bild der Deutschen in Polen und der Polen in Deutschland, Konventionen, Rituale, Verhaltensnormen, Mythen, Politik und gegenseitige Wahrnehmung. Weniger hervorgehoben wird das sprachliche Potenzial, das für die Entwicklung der interkulturellen Kompetenz notwendig ist - z. B. die interkulturelle kommunikative Kompetenz als Beherrschung des Sprachgebrauchs 
(d. h. Verwendung der Sprache-in-Funktion-in-Situation-in-Kultur vgl. House 1998: 65)

- Die Studie ergab des Weiteren eine große Diskrepanz zwischen der Selbsteinschätzung der eigenen interkulturellen Kompetenz der Studierenden und ihrer Fähigkeit, Critical-Incident-Aufgaben zu lösen. Die überwiegende Mehrheit - $80 \%$ bis $92 \%$ - traute sich einerseits $\mathrm{zu}$, interkulturell kompetent handeln zu können, aber nur ca. 20\% der Antworten auf eine Cirtical-Incident-Aufgabe lassen auf ein Verständnis der eigen/fremdkulturellen Besonderheiten im interkulturellen Kommunikationsprozess schließen. In den restlichen Fällen wird auf Vorurteile zurückgegriffen oder es wird eine ethnozentrische Interpretation angeboten.

- Die Expertenaussagen spiegeln die enthusiastische Selbsteinschätzung der Studierenden auf keinen Fall wider und decken sich in puncto Beurteilung interkultureller Kompetenz der Studierenden mit den Ergebnissen der Critical-Incident-Aufgabe. Als klar enttäuschend wird die zur Interpretation von interkulturellen Situationen benötigte Anwendung von Kulturwissen (prozedurales Wissen) sowohl über die eigene als auch über die fremde Kultur bezeichnet. Den Studierenden fällt es, so das Ergebnis, schwer, aus erlernten Fakten ein tragfähiges Interpretationsraster zu erstellen.

Als Schlussfolgerung lässt sich festhalten, dass die Fehleinschätzung eigener interkultureller Kompetenz eine profunde Unbewusstheit eigener Handlungen im Bereich der interkulturellen Kommunikation zeigt. Die Annahme eines einfachen, linearen und kontinuierlichen Transfers zwischen den affektiven, den kognitiven und den Skilldomänen ist bei den befragten polnischen Studierenden zu naiv und simplizistisch. Handlungen im interkulturellen Bereich müssen daher reflektiert und geübt werden. In Bezug auf die eigene Kultur müssen angemessene Realisierungen von (Sprech-)Handlungen reflektiert, in Bezug auf die fremde Kultur - sollten diese erlernt bzw. selber erschlossen werden. Das häufig angewandte Interpretationsraster bei der Lösung der Critical-Incident-Aufgabe (d. h. Vorurteile, Ethnozentrismen) suggeriert, dass die Probanden auf unaufwendige Interpretationsstrategien zurückgreifen.

\section{STUDIE NR. 2}

Eine qualitative Studie zur Entwicklung interkultureller und pädagogischer Kompetenz bei den Lehrenden in Schulen Niedersachsens und Sachsen-Anhalts (Rößler 2007) 
Im zweiten Fall handelt es sich um eine pädagogisch verankerte Studie zur Frage nach der Offenheit der deutschen Aufnahmegesellschaft für sprachliche und kulturelle Vielfalt und eine bi- oder multikulturelle Lebensweise. Im Mittelpunkt stehen Lehrende als Mitglieder der aufnehmenden Gesellschaft und Vertreter ihrer Institutionen), ihre Einstellungen und Haltungen, ihre Sensibilität und Bewusstheit für die Probleme einer kulturell und sprachlich heterogen zusammengesetzten Schülerschaft. Die Studie ist räumlich eingegrenzt - sie bezieht sich auf die Bundesländer SachsenAnhalt und Niedersachsen - und thematisiert die - in der Terminologie des Diskurses der Zeit ausgedrückt - Integration deutschstämmiger Aussiedlerinnen und Aussiedler aus Russland und den Staaten der ehemaligen Sowjetunion in Deutschland.

Der Untersuchung lag somit nicht der Begriff der individuellen interkulturellen Kompetenz, sondern der Offenheit der Gesellschaft (als soziales Konstrukt) zugrunde. Da die Autorin der Beherrschung der Sprache als wichtige Voraussetzung für Integration einen hohen Stellenwert zuschreibt, wird vor allem untersucht, was die Schule zum Erlernen, zur Verbesserung oder Beherrschung der Zweitsprache Deutsch leistet.

Als Datenerhebungsmethode wurden problemorientierte Interviews gewählt. Befragt wurden 20 Lehrende aus 10 Schulen aller Schulformen (Gymnasium, Realschule, Hauptschule). Der Leitfaden des Interviews entsprach der Operationalisierung der zu untersuchenden Größe und umfasste folgende Themengebiete:

- Erfahrungen mit russischsprachigen Schülerinnen und Schülern

- Situation und Bedingungen in der Schule

- Fördermöglichkeiten an der Schule

- Stellung der deutschen/russischen Sprache

- Haltungen, Einstellungen zu Aussiedlerkindern und zur Integration

- Pädagogische Veränderungen in Unterricht

- Lehreraus- und -fortbildung

- Interkulturelle Bildung und Erziehung an der Schule

Zur Auswertung der Daten wurde die Methode der Grounded Theory herangezogen. Die Analyse ergab vier typische Gruppen, von überforderten bis zu erfolgreichen interkulturell kompetenten Lehrerinnen und Lehrern. Von den strukturellen Problemen und der Überforderung mit einem sprachlich heterogenen Unterricht aufgrund fehlender Weiterbildungsmöglichkeiten sowie mangelnder methodisch-didaktischer und diagnostischer Kompetenzen von Lehrkräften im Bereich Deutsch als Zweitsprache werde ich nachstehend aus Relevanzgründen absehen.

Ich möchte hier auf einen interessanten Befund aufmerksam machen, der mir sowohl in den Interviews selbst (nachstehend in Anführungsstrichen 
zitiert) als auch in den Verallgemeinerungen von Interviewaussagen (nachstehend kursiv) aufgefallen ist und den ich hier nur exemplarisch darstelle:

[1] Die Bewältigung der sprachlich und kulturell heterogenen Unterrichtssituation fordert von der Lehrerschaft enorme Anstrengung. Diese Anforderungen erhöhen sich noch dadurch, dass Kollegen in diesem Bereich nicht ausgebildet wurden und somit mehr oder weniger autodidaktisch vorgehen (Rößler 2007: 174, Hervor. A.B.),

[2] Manchmal wïnschte ich mir, ich wäre etwas sprachbegabter und würde die Muttersprache der Schüler verstehen (ebd.: 169, Hervor. A.B.),

[3] Ursache dafür sind unter anderem die festgelegten Unterrichtsziele, die sprachlich homogenen Klassen entsprechend festgelegt sind und die Arbeit der Lehrer bestimmen. Verändert sich die Schülerschaft ohne Veränderung der Pläne, fühlen sich die Lehrer den Anforderungen nicht gewachsen und empfinden die Arbeit als schwer. Die Schüler sind dann darauf angewiesen, mit den bestehenden Bedingungen irgendwie zurechtzukommen und sich anzupassen. Die Lehrerin sieht keine Möglichkeit zu Veränderungen an der Arbeitsweise der Lehrer (ebd.: 196, Hervor. A.B.),

[4] Aber man kommt an sie [die Schüler und Schülerinnen sprachlich] ran, man muss sich eben entsprechend bemühen, dann funktioniert das auch (ebd.: 216, Hervor. A.B.).

Erstaunlich oft wird von Lehrerinnen und Lehrern im Kontext mehrsprachigen interkulturellen Unterrichts darüber berichtet, dass dieser einer kontinuierlichen Anstrengung bedarf $[1,3]$. Diese Anstrengung (neben offensichtlicher Steigerung der Kompetenzen) wird auch als Schlüssel zum Erfolg angesehen [4]. Unternimmt eine Lehrkraft diese Anstrengung nicht, so geschieht das auf Kosten der (mehrsprachigen) Schüler und Schülerinnen [3].

\section{ANSTÖSSE FÜR DIE INTERKULTURELLE (FREMDSPRACHEN-)LEHRERAUSBILDUNG}

Die Ergebnisse der zwei besprochenen Studien weisen auf eine wichtige und bisher eher unterschätze Rolle der Anstrengung in der interkulturellen Kommunikation hin. Es wird - wie belegt - nicht von der Bereitschaft gesprochen, sich auf den Fremden einzulassen, sondern von der Anstrengung, dies kontinuierlich im Alltag zu tun. Wird keine Anstrengung unternommen, Interaktionsnormen, Erwartungsmuster, kommunikative Präferenzen in einer fremden Sprache zu erlernen und diese adäquat anzuwenden, und andererseits die eigenen unaufwendigen Kommunikationsroutinen aufzugeben, so ist interkulturelle Kommunikation besonders davon bedroht, zu scheitern. Dies bezieht sich nicht nur auf Situationen, die aufgrund kultureller Unterschiede zu Krisen führen können, sondern - das bezeugt vor allem die zweite Studie - gehört generell zu einer unter Bedingungen der Interkul- 
turalität und Mehrsprachigkeit stattfindenden Kommunikation'. Mit den Anfangsbeispielen (Fall 1, Fall 2) sollte auch veranschaulicht werden, dass es sich hier nicht um eine einseitige, rein affektiv-verhaltsorientierte Fähigkeit handelt, die den angehenden Lehrerinnen und Lehrern als eine weitere Kompetenz zugemutet werden müsste. Allem voran handelt es sich bei der kommunikativen Anstrengung um eine kontinuierlich zu übende selbstreflexive (Selbst-)Beobachtungshandlung im mehrsprachigen und multikulturellen Umfeld mit eindeutigem Bezug auf die Sprachlichkeit und all das, was kommunikativ zum Ausdruck gebracht werden soll bzw. auf das gesamte Ausdrucksfeld.

Die Relevanz dessen, was hier als kommunikative Anstrengung bezeichnet wurde, ist in der Forschung bereits thematisiert worden. Hier ein von Ehlich und ten Thije in ihrer Publikation von 2010 angeführtes Beispiel, das Ähnlichkeiten zum Konzept des forschenden Lernens von House (1998) zeigt:

Es [interkulturelles kommunikatives Handeln] erfordert ein Mehr an Aufmerksamkeit und gelingt dann nicht, wenn die aus dem Bruch des Üblichen sich ergebenden Potentiale zu einer differenzierten Kommunikation nicht genutzt werden. In einer derartigen Konstellation verfestigen sich die Ungleichheiten und Ungleichzeitigkeiten der kommunikativen Ressourcen bis hin zur schließlichen Kommunikationsverweigerung (Ehlich \& ten Thije 2010: 266).

In diesem Sinne formulieren die beiden Autoren folgendes Plädoyer für die interkulturelle Bildung: „Interkulturell zu kommunizieren will also gelernt sein" (ebd.) Als Methode schlagen sie die Alltagshermeneutik vor, eine weitgehende Anbindung der Begegnung mit Anderen/Fremden an die eigene alltägliche kommunikative Praxis und die Reflexion über die dabei ablaufenden Prozesse:

Dazu gehört die Bereitschaft und Befähigung, aus Interaktionsfehlern auf eine sehr direkte Weise zu lernen und dieses Lernen produktiv zu wenden. Auch dies ist ein Kennzeichen des hermeneutischen Prozesses, das in dessen Alltagswendung von hoher Bedeutung ist (ebd.: 268).

\footnotetext{
${ }^{4}$ Es könnte weiter ausgeführt werden, dies ist generell ein Merkmal einer jeden asymmetrischen Kommunikation (z. B. zwischen Fachmann und Novizen, zwischen Fachmann und Laien usw.). In der mehrsprachigen Kommunikation wird die Asymmetrie oft durch die Wahl einer gemeinsamen Lingua franca reduziert. Dies ist allerdings meistens der Fall in den Geschäftsbeziehungen (z. B. beweist Kiefer (2013), dass in der deutsch-polnischen Wirtschaftskommunikation trotz relativ guter Deutschkenntnisse der polnischen Geschäftspartner als Kommunikationssprache meistens Englisch gewählt wird) und kann nicht in jeder Situation frei gewählt werden (bzw. es ist die Gesprächspartner nicht sinnvoll, diese Strategie anzuwenden).
} 
Sowohl Fremdsprachendidaktik als auch Zweitsprachendidaktik und Pädagogik verfügen über Verfahren, die es Studierenden ermöglichen, in ihrer akademischen Tätigkeit jene linguistisch orientierte Alltagshermeneutik zu praktizieren. Das heißt, jene kommunikative Anstrengung zu unternehmen, diese zu reflektieren und aus dieser zu lernen. Mögliche Verfahren sind z. B.:

- Sprachbiographien (Krumm 2010; Franceschini 2004; Fix 1993)

- Lernertagebücher (Bailey 1983)

- Ethnographische Feldnotizen (House 1998)

- Teilnehmende Beobachtung (Pilch \& Bauman 2001; Spradley 1979)

Die Verfahren gestatten es, persönlich erlebte kommunikative Situationen zu dokumentieren und unter Einsatz linguistischen Wissens zu interpretieren. Als Analyseinstrumentarium würde sich eine Beschreibung und Interpretation der jeweiligen diskursiven Strukturen nach den Prinzipien der funktionalen Pragmatik anbieten, die von Jan ten Thije (2002) für die Interpretation von interkulturellen Diskursen adaptiert wurde.

Die eingangs vorgestellten Fallbeispiele, die aus meiner eigenen Sammlung von Feldnotizen stammen, können zeigen, wie diese alltagspraktische Reflexion über sprachliche Kommunikation umzusetzen und systematisch zu betreiben wäre. Gelingt es, dieses forschende Lernen auch in Curricula für Lehramtsstudierende umzusetzen, dann ist eine der besten Voraussetzungen für lebenslanges eigenes Weiterlernen geschaffen, auch für diejenigen Lehrerinnen und Lehrer, die in einem multilingualen Umfeld arbeiten und fähig sein müssen, den monolingualen Habitus (Gogolin 1994) der Schule zu überwinden.

\section{LITERATURVERZEICHNIS}

Agar, M. H. (1994). Language shock: Understanding the culture of conversation. New York: William Morrow.

Bailey, K. M. (1983). Competitiveness and anxiety in adult second language learning: Looking at an through the diary studies. In: H. W. Seliger, / M. H. Long (Hrsg.), Classroom oriented research in the second language acquisition (S. 67-103). Rowley: Newbury House.

Błażek, A. (2008). Evaluation interkultureller Kompetenz bei angehenden Deutschlehrerinnen und -lehrern in Polen. Poznań: Wydawnictwo Naukowe UAM.

Błażek, A. (2018). Einheit in Vielfalt: der Bologna-Prozess. Fachlexikologische und fachkommunikative Aspekte. Berlin: Frank \& Timme.

Byram, M. (1997). Teaching and assessing intercultural communicative competence. Clevedon: Multicultural Matters.

Clark, H. H. (1996). Using language. Cambridge: University Press.

Ehlich, K. / ten Thije, J. D. (2010). Linguistisch begründete Verfahren der Analyse interkultureller Kommunikation. In: A. Weidemann / J. Straub / S. Nothnagel (Hrsg.), Wie lehrt man interkulturelle Kompetenz. Theorien, Methoden und Praxis in der Hochschulausbildung. Ein Handbuch (S. 265-285). Bielefeld: transcript Verlag. 
Fix, U. (1993). Sprachbiographien. Der deutsch-deutsche Sprachgebrauchswandel im Erinnern und Erleben von Zeitzeugen. In: I. Gamer-Wallert / E. Blumenthal / G. Klinger (Hrsg.), Nähe und Ferne. Erlebte Geschichte im geteilten und vereinigten Deutschland (S. 78-95). Tübingen: Attempto.

Franceschini, R. (2004). Sprachbiographien: das Basel-Prag-Projekt (BPP). In: R. Franceschini / J. Miecznikowski (Hrsg.), Leben mit mehreren Sprachen. Vivre avec plusieurs langues (S. 121-145). Bern, Berlin et. al.: Peter Lang.

Garfinkel, H. (1967). Studies in Ethnomethodology. Cambridge: Polity Press.

Garfinkel, H. (2006 [1948]). Seeing Sociologically. The Routine Grounds of Social Action. Lanham: Rowman \& Littlefield.

Gogolin, I. (1994). Der monolinguale Habitus der multilingualen Schule. Münster: Waxmann.

House, J. (1998). Kontrastive Pragmatik und interkulturelle Kompetenz im Fremdsprachenunterricht. In: K.-D. Baumann / H. Kalverkämper (Hrsg.), Kontrast und Äquivalenz. Beiträge zu Sprachvergleich und Übersetzung (S. 63-88). Tübingen: Narr.

Kiefer, K.-H. (2013). Kommunikative Kompetenzen im Berufsfeld der internationalen Steuerberatung. Frankfurt am Main: Peter Lang.

Krumm, H.-J. (2010). Mehrsprachigkeit in Sprachenporträts und Sprachenbiographien von Migrantinnen und Migranten. Arbeitskreis Deutsch als Fremdsprache (AkDaf) Rundbrief 61, 16-24.

Niemierko, B. (1999). Pomiar wyników ksztatcenia. Warszawa: WSiP.

Pilch, T. / Bauman, T. (2001). Zasady badań pedagogicznych. Warszawa: Wydawnictwo Akademickie "Żak".

Rößler, S. (2007). Eine qualitative Studie zur Entwicklung interkultureller und pädagogischer Kompetenz bei den Lehrern in Schulen Niedersachsens und Sachsen-Anhalts. Magdeburg: Docupoint.

Sercu, L. (2004). Assessing intercultural competence: a framework for systematic test development in foreign language education and beyond. Intercultural Education, 15 (1), 73-89.

Spradley, J. P. (1979). The ethnographic interview. New York: Holt, Rinehart and Winston.

ten Thije, J. (2002). Stufen des Verstehens bei der Interpretation von interkulturellen Diskursen. In: H. Kotthoff (Hrsg.), Kultur(en) im Gespräch (S. 61-98). Tübingen: Gunter Narr Verlag.

Received: 12.08 .2018 ; revised: 23.08 .2018 
